\title{
Magnon Decay in Gapped Quantum Spin Systems
}

\section{Citation}

Kolezhuk, Alexei, and Subir Sachdev. 2006. "Magnon Decay in Gapped Quantum Spin Systems." Physical Review Letters 96 (8). https://doi.org/10.1103/physrevlett.96.087203.

\section{Permanent link}

http://nrs.harvard.edu/urn-3:HUL.InstRepos:41417305

\section{Terms of Use}

This article was downloaded from Harvard University's DASH repository, and is made available under the terms and conditions applicable to Other Posted Material, as set forth at http:// nrs.harvard.edu/urn-3:HUL.InstRepos:dash.current.terms-of-use\#LAA

\section{Share Your Story}

The Harvard community has made this article openly available.

Please share how this access benefits you. Submit a story.

Accessibility 


\title{
Magnon decay in gapped quantum spin systems
}

\author{
Alexei Kolezhuk* and Subir Sachdev \\ Department of Physics, Harvard University, Cambridge MA 02138
}

\begin{abstract}
In the $\mathrm{O}(3) \sigma$-model description of gapped spin systems, $S=1$ magnons can only decay into three lower energy magnons. We argue that the symmetry of the quantum spin Hamiltonian often allows decay into two magnons, and compute this decay rate in model systems. Two magnon decay is present in Haldane gap $S=1$ spin chains, even though it cannot be induced by any allowed term written in powers and gradients of the $\sigma$-model field. We compare our results with recent measurements of Stone et al. on a two-dimensional spin system.
\end{abstract}

PACS numbers: 75.10.Jm, 75.40.Gb

Introduction.- Stone et al $1 \frac{1}{1}$ have recently studied the spectrum of the $S=1$ magnon excitations of the gapped quasi-two-dimensional spin dimer compound $\left(\mathrm{C}_{4} \mathrm{H}_{12} \mathrm{~N}_{2}\right) \mathrm{Cu}_{2} \mathrm{Cl}_{6}$ (piperazinium hexachlorodicuprate, PHCC). They observed that the magnons become unstable when their energy crosses the lower boundary of the two-magnon continuum, and measured magnon lifetimes. These observations are interesting because, at first glance, they appear to be in conflict with the standard phenomenological description of low energy $S=1$ excitations in a confining spin-gap systems. This model 2.3 is expressed in terms of a field $\mathbf{n}(x, \tau)$, where $x$ is a $d$-dimensional spatial coordinate, $\tau$ is imaginary time, and $\mathbf{n}$ is three-component vector in spin space. Using Landau-Ginzburg arguments, the effective action for the magnons, near the minimum of their dispersion, can be written in the form

$\mathcal{S}_{\boldsymbol{n}}=\int d^{d} x d \tau\left\{\frac{1}{2 g}\left(\left(\partial_{\tau} \boldsymbol{n}\right)^{2}+c^{2}\left(\partial_{x} \boldsymbol{n}\right)^{2}\right)+\frac{u}{2}\left(\boldsymbol{n}^{2}-\alpha\right)^{2}\right\}$

Harmonic quantum fluctuations of $\boldsymbol{n}$ about $\boldsymbol{n}=0$ in a renormalized effective potential then constitute the triply degenerate magnon in the spin-gap state. In this picture, it is clear from the quartic non-linearity in $\mathcal{S}_{\boldsymbol{n}}$ that this magnon can only decay into three magnons, and decay into two magnons is prohibited.

It is useful to recall the constraints imposed by symmetry more carefully for the familiar case of a spin $S$ antiferromagnetic chain $(d=1)$. This model has spin rotation symmetry, time reversal symmetry (under which $\tau \rightarrow-\tau$ and $\boldsymbol{n} \rightarrow-\boldsymbol{n}$ ), reflection symmetry about sites (under which $x \rightarrow-x$ and $\boldsymbol{n} \rightarrow \boldsymbol{n}$ ), and reflection symmetry about centers of bonds (under which, in the continuum limit, $x \rightarrow-x$ and $\boldsymbol{n} \rightarrow-\boldsymbol{n})$. These symmetries are very restrictive and only allow a single term with three powers of $\boldsymbol{n}$ : the topological ' $\theta$-term' ${ }^{2}$

$$
\mathcal{S}_{\theta}=i \theta \int d x d \tau \boldsymbol{n} \cdot\left(\partial_{x} \boldsymbol{n} \times \partial_{\tau} \boldsymbol{n}\right),
$$

in the fixed length limit $u \rightarrow \infty$ with $\alpha=1$ and $\theta$ quantized at $\theta=S / 2$. In this limit the integrand in $\mathcal{S}_{\theta}$ is quantized in integer multiples of $4 \pi$, and only then is $e^{-\mathcal{S}_{\theta}}=1$ invariant under all symmetries noted above. However, we are interested in the nature of the non-linear terms as they act on the fully renormalized quasiparticles of the gapped antiferromagnet with integer $S$. As noted above, these can be considered to be harmonic fluctuations of a renormalized field $\boldsymbol{n}$ about $\boldsymbol{n}=0$, and the amplitude of this renormalized field is not constrained to be unity. For such a field $\boldsymbol{n}$, it is easy to see that there is no term with three powers of $\boldsymbol{n}$, and arbitrary spatial and temporal gradients which is invariant under the symmetries.

Here we will examine the issue of two magnon decay using a lattice formulation. Our analysis begins below with a microscopic Hamiltonian appropriate for PHCC. We will compute the lifetime due to decay into two magnons, and compare our results with the observations of Stone $e t$ $a l \underline{1}$ The confrontation between theory and experiments places new constraints on the values of the microscopic exchange constants. We then show that similar processes also exist for a generic $S=1$ spin chain, and are consistent with all symmetries of the lattice Hamiltonian; this is the case even though such processes do not appear in any perturbation of the continuum theory.

Magnon lifetimes in $\mathrm{PHCC}$. - The $\mathrm{Cu}^{2+}$ spins in PHCC form a lattice of dimers in the $(a c)$ crystalline plane as shown in Fig. 11. We will denote the interdimer exchange interactions as $J_{\delta}^{\sigma \sigma^{\prime}}$, where $\sigma, \sigma^{\prime}=1,2$ label two spins inside a dimer and the vector $\boldsymbol{\delta}=(m, n) \equiv m \widehat{\boldsymbol{a}}+n \widehat{\boldsymbol{c}}$ connects the dimer centers. For a description of such systems it is convenient to use the so-called bond-boson formalism ${ }^{4}$ where spin- $\frac{1}{2}$ operators $\boldsymbol{S}_{1}, \boldsymbol{S}_{2}$ at each dimer (see Fig. 1) are represented in terms of three hardcore bosons $t_{\alpha}, \alpha=(x, y, z)$ which correspond to three excited triplet states of the dimer:

$$
S_{1,2}^{\alpha}= \pm \frac{1}{2}\left(t_{\alpha}+t_{\alpha}^{\dagger}\right)+\frac{1}{2} i \varepsilon_{\alpha \beta \gamma} t_{\beta} t_{\gamma}^{\dagger} .
$$

If one assumes the isotropic Heisenberg coupling between dimers, the spin Hamiltonian takes the form $\widehat{H}=\widehat{H}_{0}+{ }_{2}^{H}$ $+\widehat{H}_{3}+\widehat{H}_{4}$, where $\widehat{H}_{0}=J_{0} \sum_{\boldsymbol{r}} t_{\boldsymbol{r} \alpha}^{\dagger} t_{\boldsymbol{r} \alpha}$ is determined by the intradimer exchange $J_{0}$, and $\widehat{H}_{2,3,4}$ correspond to the interdimer interaction. (The index $\boldsymbol{r}$ here labels the sites of the lattice formed by dimers).

The quadratic part of the interdimer Hamiltonian

$$
\widehat{H}_{2}=\frac{1}{2} \sum_{\left(\boldsymbol{r} \boldsymbol{r}^{\prime}\right)} J_{\boldsymbol{r}-\boldsymbol{r}^{\prime}}^{\mathrm{eff}}\left(t_{\boldsymbol{r} \alpha}^{\dagger} t_{\boldsymbol{r}^{\prime} \alpha}+t_{\boldsymbol{r} \alpha} t_{\boldsymbol{r}^{\prime} \alpha}+\text { h.c. }\right),
$$


as well as the four-particle interaction $\widehat{H}_{4}$,

$$
\widehat{H}_{4}=\frac{1}{2} \sum_{\left(\boldsymbol{r} \boldsymbol{r}^{\prime}\right)} J_{\boldsymbol{r r}^{\prime}}^{\mathrm{eff}}\left\{t_{\boldsymbol{r} \alpha}^{\dagger} t_{\boldsymbol{r}^{\prime} \alpha}^{\dagger} t_{\boldsymbol{r} \beta} t_{\boldsymbol{r}^{\prime} \beta}-t_{\boldsymbol{r}^{\prime} \alpha}^{\dagger} t_{\boldsymbol{r} \beta}^{\dagger} t_{\boldsymbol{r} \alpha} t_{\boldsymbol{r}^{\prime} \beta}\right\},
$$

depend only on specific combinations $J_{\boldsymbol{r}-\boldsymbol{r}^{\prime}}^{\text {eff }}$ of exchange couplings between the dimers located at sites $\boldsymbol{r}$ and $\boldsymbol{r}^{\prime}$,

$$
J_{\boldsymbol{r}-\boldsymbol{r}^{\prime}}^{\mathrm{eff}}=\frac{1}{2}\left(J_{\boldsymbol{r}-\boldsymbol{r}^{\prime}}^{11}+J_{\boldsymbol{r}-\boldsymbol{r}^{\prime}}^{22}-J_{\boldsymbol{r}-\boldsymbol{r}^{\prime}}^{12}-J_{\boldsymbol{r}-\boldsymbol{r}^{\prime}}^{21}\right) .
$$

The three-magnon term $\widehat{H}_{3}$ depends on different combinations of exchange constants $F_{\boldsymbol{r}-\boldsymbol{r}^{\prime}}$ that are nonzero only if the interdimer coupling is not symmetric with respect to the interchange of the spin indices 1 and 2 :

$$
\begin{aligned}
& \widehat{H}_{3}=\frac{i}{2} \sum_{\left(\boldsymbol{r} \boldsymbol{r}^{\prime}\right)} \varepsilon_{\alpha \beta \gamma} F_{\boldsymbol{r}-\boldsymbol{r}^{\prime}}\left(t_{\boldsymbol{r} \alpha}^{\dagger} t_{\boldsymbol{r}^{\prime} \beta}^{\dagger} t_{\boldsymbol{r}^{\prime} \gamma}-t_{\boldsymbol{r}^{\prime} \alpha}^{\dagger} t_{\boldsymbol{r} \beta}^{\dagger} t_{\boldsymbol{r} \gamma}\right)+\text { h.c. } \\
& F_{\boldsymbol{r}-\boldsymbol{r}^{\prime}}=\frac{1}{2}\left(J_{\boldsymbol{r}-\boldsymbol{r}^{\prime}}^{22}-J_{\boldsymbol{r}-\boldsymbol{r}^{\prime}}^{11}-J_{\boldsymbol{r}-\boldsymbol{r}^{\prime}}^{12}+J_{\boldsymbol{r}-\boldsymbol{r}^{\prime}}^{21}\right)
\end{aligned}
$$

This type of interaction arises generically in the theory; however, it is usually neglected in practical calculations.

If one neglects magnon interaction completely, taking into account only the quadratic part of the Hamiltonian $\widehat{H}_{0}+\widehat{H}_{2}$, and uses this approximation to describe the experimentally observed magnon dispersion and to find out the microscopic exchange couplings, one can obtain estimates for the intradimer exchange constant $J_{0}$ and for "effective couplings" $J_{\boldsymbol{r}-\boldsymbol{r}^{\prime}}^{\text {eff }}$ only. The individual couplings $J_{\boldsymbol{r}-\boldsymbol{r}^{\prime}}^{11}, J_{\boldsymbol{r}-\boldsymbol{r}^{\prime}}^{12}$, etc. cannot be determined in this noninteracting magnon approximation that is commonly used by experimentalists for the description of the dispersion data (see, e.g., Refs. 5 6.7).

Since an analytical treatment of the full interacting Hamiltonian for realistic models does not seem feasible, one has to resort to extensive numerical calculations to extract the coupling information from the dispersion data. Such calculations based on cluster expansions were actually performed for various spin dimer materials, $; .9 .10$ however, even in this approach the problem remains difficult because for weak interdimer interactions the magnon dispersion is rather insensitive to the variations of the individual exchange couplings which keep the "effective" interactions $J_{\boldsymbol{r}-\boldsymbol{r}^{\prime}}^{\text {eff }}$ constant.

The magnon dispersion in PHCC was studied experimentally in Ref. 5; the spin excitations were found to have a spectral gap $\Delta \approx 1 \mathrm{meV}$ and a bandwidth of about $1.8 \mathrm{meV}$. It was shown that the observed spectrum is well described within the noninteracting magnon approximation, if one assumes the existence of effective interdimer interactions $J_{\delta}^{\text {eff }}$ with $\boldsymbol{\delta}=(1,0),(0,1),(1,1),(1,-1)$, $(2,0)$, and $(0,2)$, with $J_{0} \simeq 2.33 \mathrm{meV}, J_{(1,0)}^{\text {eff }} \simeq 0.44 \mathrm{meV}$, $J_{(0,1)}^{\text {eff }} \simeq 0.23 \mathrm{meV}, J_{(1,1)}^{\mathrm{eff}} \simeq J_{(1,-1)}^{\mathrm{eff}} \simeq-0.084 \mathrm{meV}$, $J_{(2,0)}^{\text {eff }} \simeq-0.073 \mathrm{meV}$, and $J_{(0,2)}^{\text {eff }} \simeq-0.047 \mathrm{meV} \stackrel{11}{\underline{11}}$ this approximation, the magnon dispersion takes the form

$$
\varepsilon(\boldsymbol{k})=\left\{J_{0}^{2}+2 J_{0} B_{\boldsymbol{k}}\right\}^{1 / 2}, \quad B_{\boldsymbol{k}}=\sum_{\boldsymbol{\delta}} J_{\boldsymbol{\delta}}^{\mathrm{eff}} \cos (\boldsymbol{k} \cdot \boldsymbol{\delta})
$$

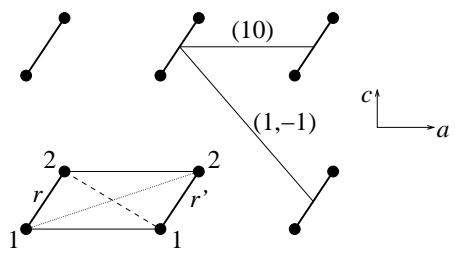

FIG. 1: A schematic view of the coupled spin dimer structure in the crystallographic $(a c)$ plane of PHCC, and the notation used. Two spins in each dimer are distinguished by the spin index $\sigma=1,2$, and each dimer is labeled by the position of its center $\boldsymbol{r}$. Exchange couplings between individual spins are then denoted as $J_{\boldsymbol{r}-\boldsymbol{r}^{\prime}}^{\left(\sigma \sigma^{\prime}\right)}$. In PHCC, the most important exchange links are given by $\boldsymbol{r}-\boldsymbol{r}^{\prime}=(1,0),(0,1),(1,1),(1,-1)$, and possibly also $(2,0)$ and $(0,2)$ (in lattice units).

and the quasiparticle operators $b_{\boldsymbol{k}}$ are connected to the dimer triplet-creating operators $t_{\boldsymbol{k}}$ through the standard Bogoliubov transformation $t_{k}=u_{k} b_{k}+v_{k} b_{-k}^{\dagger}$, where the coefficients $u_{k}=\cosh x_{k}, v_{k}=\sinh x_{k}$, with $\tanh \left(2 x_{\boldsymbol{k}}\right)=-B_{\boldsymbol{k}} /\left(J_{0}+B_{\boldsymbol{k}}\right)$ and $\operatorname{sign}\left(v_{\boldsymbol{k}}\right)=-\operatorname{sign}\left(B_{\boldsymbol{k}}\right)$.

The four-magnon interaction term $\widehat{H}_{4}$ leads in fact to a renormalization of the amplitudes $J_{\delta}^{\text {eff }}$ in $\widehat{H}_{2}$ and can be ignored as far as one uses the values of the effective couplings $J_{\delta}^{\text {eff }}$ obtained from a comparison with the experimental data, since the above renormalization is already included in this way. The same argument applies to the hardcore constraint which, for low density of virtual triplet pairs, in the leading approximation can be shown just to renormalize the self-energy of a magnon ${ }^{12}$

The three-magnon term $\widehat{H}_{3}$ does not contribute to the dispersion in the first order of the perturbation theory, and its main physical effect is to produce an instability of magnons with energies above the two-particle continuum threshold. In terms of transformed quasiparticle operators $b_{\boldsymbol{k}}$ this interaction can be written as

$$
\begin{aligned}
\widehat{H}_{3} & =\sum_{(x y z)} \sum_{12} M_{12} b_{1+2, z}^{\dagger} b_{1, x} b_{2, y}+\cdots+\text { h.c. }, \\
M_{12} & =\sum_{\boldsymbol{\delta}} F_{\boldsymbol{\delta}}\left\{\left(u_{1+2}-v_{1+2}\right)\left(v_{1} u_{2}-v_{2} u_{1}\right)\right. \\
& \times \sin \left(\boldsymbol{k}_{1} \cdot \boldsymbol{\delta}+\boldsymbol{k}_{2} \cdot \boldsymbol{\delta}\right) \\
& -\left(u_{1}-v_{1}\right)\left(u_{1+2} u_{2}+v_{1+2} v_{2}\right) \sin \left(\boldsymbol{k}_{1} \cdot \boldsymbol{\delta}\right) \\
& \left.+\left(u_{2}-v_{2}\right)\left(u_{1+2} u_{1}+v_{1+2} v_{1}\right) \sin \left(\boldsymbol{k}_{2} \cdot \boldsymbol{\delta}\right)\right\},
\end{aligned}
$$

where $(x y z)$ denotes a cyclic summation over the triplet component indices, $(1,2) \equiv \boldsymbol{k}_{1,2}$, and the remaining terms describing three-particle creation are omitted for clarity. The decay rate $\Gamma(\boldsymbol{p})$ of a magnon with the wave vector $\boldsymbol{p}$ according to the Fermi golden rule is

$$
\Gamma(\boldsymbol{p})=2 \pi \sum_{\boldsymbol{k}}\left|M_{\boldsymbol{k}, \boldsymbol{p}-\boldsymbol{k}}\right|^{2} \delta(\varepsilon(\boldsymbol{p})-\varepsilon(\boldsymbol{k})-\varepsilon(\boldsymbol{p}-\boldsymbol{k})) .
$$

After performing the delta-function integration, the latter expression is reduced to a one-dimensional quadrature which can be computed numerically. 


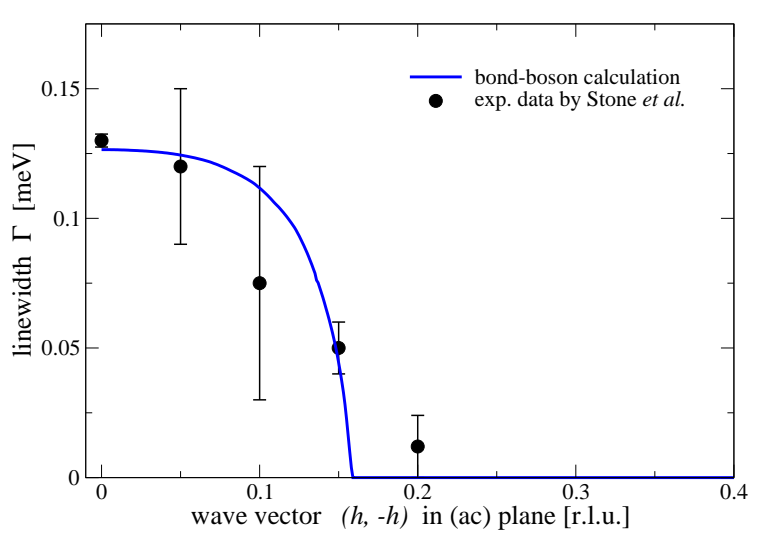

FIG. 2: Magnon linewidth $\Gamma(\boldsymbol{p})$ in PHCC as a function of the wave vector $\boldsymbol{p}=(h,-h)$ [r.l.u.] in the $(a c)$ plane. The solid line displays the result of the bond-boson calculation (see text) and the symbols correspond to experimental data of Stone et al. ${ }^{1}$

In Ref. 9 the magnon dispersion in PHCC was analyzed on the basis of the fourth-order cluster expansion calculation, and it was concluded that a good fit to the data of Ref. 5 can be achieved even without including the links with $\boldsymbol{\delta}=(2,0)$ or $(0,2)$ (for those latter links, the existence of exchange paths seems questionable). Since we are not taking into account higher-order effects, we will adopt the coupling values $J_{\delta}^{\text {eff }}$ proposed in Ref. 5 for the sake of consistency; one may argue that this is not going to introduce any serious problem since the role of $J_{\delta}^{\text {eff }}$ in our calculation here is just to mimic the correct behavior of $\varepsilon(\boldsymbol{k})$ and the Bogoliubov factors $u_{\boldsymbol{k}}, v_{\boldsymbol{k}}$.

Another conclusion of Ref. 9 was that the links with $\boldsymbol{\delta}=(1,1)$ and $(1,-1)$ are fully due to the "diagonal" couplings of the $J^{12}$ and $J^{21}$ type, respectively, while the other interdimer links have symmetric couplings in the sense that $J_{\delta}^{12}=J_{\delta}^{21}$ and $J_{\delta}^{11}=J_{\delta}^{22}$, so that all $F_{\delta}=0$ except $F_{(1,-1)} \approx-F_{(1,1)} \approx 0.09 \mathrm{meV}$.

We have fitted the experimental data 1 for $\Gamma$ as a function of the wave vector $\boldsymbol{p}$ in the (ac) plane, using the amplitudes $F_{\delta}$ as fitting parameters. If one assumes that all the individual exchange couplings are antiferromagnetic, a natural constraint arises that the absolute value of the amplitude $F_{\delta}$ cannot exceed the absolute value of the corresponding $J_{\delta}^{\text {eff }}$. It turns out that the observed magnitude of the linewidth ${ }^{1}$ (the maximum of $\Gamma \simeq 0.13 \mathrm{meV}$ is reached at $\boldsymbol{p}=(0,0))$ cannot be achieved if one adopts the coupling pattern proposed in Ref. 9: the contribution of $F_{(1,1)}$ and $F_{(1,-1)}$ alone is definitely insufficient (with $\Gamma(0)$ one order of magnitude below the observed value). The linewidth is rather insensitive to the value of $F_{(0,1)}$ : even at $F_{(0,1)}=J_{(0,1)}^{\text {eff }}$ the corresponding contribution to $\Gamma(\boldsymbol{p}=0)$ is of the order of $10^{-4} \mathrm{meV}$. Similarly, inclusion of longer links like $F_{(2,0)}$ and $F_{(0,2)}$ does not help, since at their "full strength" (i.e., at $F_{\delta}=J_{\delta}^{\text {eff }}$ ) they yield the contributions to $\Gamma(0)$ of the order of several $\mu \mathrm{eV}$. The only remaining way to explain the observed values of $\Gamma$ is to assume that $F_{(10)} \neq 0$; indeed, as shown in Fig. 2 a reasonable fit of the experimental data is achieved with $F_{(1,0)}=0.7 J_{(1,0)}^{\mathrm{eff}}, F_{(1,1)}=-J_{(1,1)}^{\mathrm{eff}}$, and $F_{(1,-1)}=J_{(1,-1)}^{\mathrm{eff}}$. The shown fit is not unique: the signs of $F_{\delta}$ were chosen to provide the maximum enhancement of the total amplitude of the matrix element $M_{12}$, and the amplitudes $F_{(2,0)}$ and $F_{(0,2)}$ for "questionable" links were dropped altogether, so one should view this result as a sort of "lower bound" for $F_{(1,0)}$. Nevertheless, our results are a clear indication that the interdimer interactions along the $(1,0)$ link in the $(a c)$ plane are asymmetric with respect to the spin index interchange $1 \leftrightarrow 2$. It is worth noting that the signs of $F_{(1,1)}$ and $F_{(1,-1)}$ agree with the pattern proposed in Ref. 9 .

In PHCC, the crystal symmetry makes all $J^{11}$ and $J^{22}$ equal, so the only asymmetry giving rise to nonzero amplitudes $F_{\delta}$ can be the difference between $J_{\delta}^{12}$ and $J_{\delta}^{21}$. The existence of a large $F_{\boldsymbol{\delta}}$ for the strongest $(1,0)$ link implies that the magnitudes of the individual couplings $J_{(1,0)}^{\sigma \sigma^{\prime}}$ are considerably larger than the effective "dimer" exchange constant $J \equiv J_{(1,0)}^{\text {eff }} \simeq 0.44 \mathrm{meV}$. For instance, if one assumes for simplicity that $J_{(1,0)}^{12}=0$ then $J_{(1,0)}^{11}=J_{(2,0)}^{22} \simeq 1.7 J$ and $J_{(1,0)}^{21} \simeq 1.4 J ;$ another, although less likely possibility is that the "diagonal" couplings have different signs, $J_{(1,0)}^{12}<0$ and $J_{(1,0)}^{21}>0$.

Magnon decay in $S=1$ Haldane chains.- Let us now turn to the $S=1$ spin chain. Indeed, this model can also be viewed as a chain of $S=\frac{1}{2}$ dimers with antiferromagnetic intradimer exchange $4 J$ and an additional local constraint symmetrizing the edge spins of every two neighboring dimers, and thus coupling them into an effective spin-1, see Fig. 3. This constraint can be taken into account 'on average' at a mean-field level by adding the Lagrange multiplier term of the form $-\mu \sum_{n}\left(\boldsymbol{S}_{1, n+1} \cdot \boldsymbol{S}_{2, n}-\frac{1}{4}\right)$, playing the role of a ferromagnetic "Hund's rule" interdimer interaction which has to be determined self-consistently. It is then obvious that the interaction between the dimers is asymmetric and the effective Hamiltonian will contain the term of the form (5) with a single $F_{1}=\frac{1}{2} \mu$. It is easy to see that this term is consistent with all symmetries of the initial Hamiltonian, e.g., it satisfies the reflection symmetry about the $n_{0}$-th bond, which in the bond-boson language corresponds to the transformation $t_{n, \alpha} \mapsto-t_{2 n_{0}-n, \alpha}$, as well as the symmetry of reflection about the $n_{0}$-th "site" (i.e., the interdimer bond to the right of the $n_{0}$-th dimer, see Fig. (3)), which corresponds to $t_{n, \alpha} \mapsto-t_{2 n_{0}+1-n, \alpha}$.

Then, a simple golden rule calculation yields the magnon linewidth in the Haldane chain as a function of the its momentum $p$ :

$$
\Gamma(p)=\left|M_{k_{0}, p-k_{0}}\right|^{2} /\left|\varepsilon_{k_{0}}^{\prime}-\varepsilon_{p-k_{0}}^{\prime}\right|,
$$

where $k_{0}$ is the solution of the equation $\varepsilon_{k_{0}}+\varepsilon_{p-k_{0}}=\varepsilon_{p}$, and $\varepsilon_{k}=2\left(4 J^{2}+\mu J \cos k\right)^{1 / 2}$ is the single-magnon dispersion. Using the known value of the Haldane gap $\Delta \simeq 0.4 J$, one can fix the renormalized value of $\mu$ to be slightly below $4 J$. According to (5), the matrix ele- 


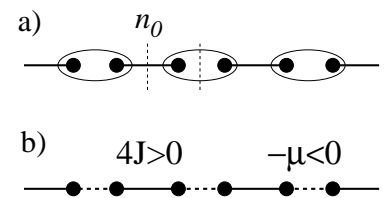

FIG. 3: a) representation of spin-1 Haldane chain as a spin- $\frac{1}{2}$ chain with symmetrized spin- $\frac{1}{2}$ degrees of freedom (denoted by the ovals); b) at the mean-field level the symmetrization is equivalent to a ferromagnetic "Hund's rule" interaction $-\mu$.

ment has the structure $M_{k, p-k}=f_{p}(k)-f_{p}(p-k)$, so the linewidth becomes zero at the wave vector threshold $p=p_{c}$, defined by $\varepsilon\left(p_{c}\right)=2 \varepsilon\left(p_{c} / 2\right)$ (here $\left.p_{c} \in[\pi, 2 \pi]\right)$; it is exactly the point of crossing with the the two-particle continuum. Above the continuum threshold, in the vicinity of $p_{c}$ the linewidth grows as a square root:

$$
\Gamma(p) \simeq \frac{2\left|f^{\prime}\left(p_{c} / 2\right)\right|^{2}}{\left|\varepsilon^{\prime \prime}\left(p_{c} / 2\right)\right|}\left|\frac{\varepsilon^{\prime}\left(p_{c}\right)-\varepsilon^{\prime}\left(p_{c} / 2\right)}{\varepsilon^{\prime \prime}\left(p_{c} / 2\right)}\right|^{1 / 2} \sqrt{p-p_{c}} .
$$

It is notable that two-magnon decay processes, strictly forbidden in the Haldane chain within the effective continuum field theory, nevertheless survive in the full lattice description.

Using the above simple estimate for $\mu$, one can see that the magnon linewidth in the Haldane chain grows fast to rather large values, e.g., at $p=0$ one obtains $\Gamma \simeq 3.4 J$, to be compared to $\varepsilon(p=0) \simeq 5.6 \mathrm{~J}$. Such a large value of the linewidth arises because the effective "interdimer" interaction $\mu$ is not small compared to the "intradimer" coupling $4 J$. An experimental observation in that case would show a very fast disappearance of quasiparticle excitation above the continuum threshold, similar to the picture observed recently ${ }^{13}$ in a spin ladder compound $\left(\mathrm{CH}_{3}\right)_{2} \mathrm{CHNH}_{3} \mathrm{CuCl}_{3}$. However, one should bear in mind that the behavior of linewidth in the vicinity of the threshold depends on the details of the wave vector dependence of the matrix element, which cannot be accounted for in the simple qualitative description sketched above: for instance, if $f^{\prime}\left(p_{c} / 2\right)$ is anomalously small for some reason, then one has to take into account higher derivatives of $f(p)$ and (10) gets replaced by a sum of competing terms, $\Gamma(p) \propto \sum_{n=1} A_{n}\left(p-p_{c}\right)^{n}$. Recently, a finite magnon linewidth slowly emerging at $p_{c} \simeq 0.6 \pi$ was observed ${ }^{14}$ in the $S=1$ chain material $\mathrm{CsNiCl}_{3}$. It would be interesting if future experiments could provide more information on two-magnon decay in Haldane spin chain.

Summary.- To conclude, we have shown that elementary excitations in a gapped dimerized spin system can become unstable above the two-particle continuum threshold, and that the dependence of the magnon linewidth on the wave vector can be used as an additional input (complementing the magnon dispersion) to extract the information on the exchange interactions pattern. On the basis of fitting the recent results 1 on the magnon linewidth in the quasi-two-dimensional spin dimer material PHCC, we conclude that interdimer couplings along the $a$ axis in this material are strongly asymmetric, contrary to the previously assumed ${ }^{9}$ pattern. We argue that three-magnon interaction, which makes possible the instability of a single-magnon excitation against the decay into two particles, is generically present in other gapped spin systems such as spin-1 Haldane chains.

Acknowledgments.- We thank C. Broholm, T. Senthil, and I. Zaliznyak for useful discussions. This research was supported by NSF Grant DMR-0537077. AK is supported by the Heisenberg Program Grant No. KO 2335/1-1 from Deutsche Forschungsgemeinschaft.
* On leave from the Institute of Magnetism, Academy of Sciences and Ministry of Education, 03142 Kiev, Ukraine

1 M. B. Stone, I. A. Zaliznyak, T. Hong, C. L. Broholm, and D. H. Reich, cond-mat/0511266

2 F. D. M. Haldane, Phys. Rev. Lett. 50, 1153 (1983).

3 I. Affleck, Phys. Rev. B 43, 3215 (1991).

4 S. Sachdev and R. N. Bhatt, Phys. Rev. B 41, 9323 (1990)

5 M. B. Stone, I. Zaliznyak, D. H. Reich, and C. Broholm, Phys. Rev. B 64, 144405 (2001).

${ }^{6}$ B. Leuenberger, A. Stebler, H. U. Güdel, A. Furrer, R. Feile, and J. K. Kjems, Phys. Rev. B 30, 6300 (1984).

7 T. Kato, K. Takatsu, H. Tanaka, W. Shiramura, M. Mori, K. Nakajima, K. Kakurai: J. Phys. Soc. Japan 67, 752 (1998).

8 M. Müller and H.-J. Mikeska, J. Phys.: Cond. Matter 13,
7633 (2000).

9 H.-J. Mikeska and M. Müller, Appl. Phys. A 74, S580 (2002).

10 A. Oosawa, T. Kato, H. Tanaka, K. Kakurai, M. Muller, and H.-J. Mikeska, Phys. Rev. B 65, 094426 (2002).

11 The constants $B_{0}, B_{\delta}$ given in Table II of Ref. [5] are connected to our notation through $B_{0}=J_{0}^{2}, B_{\delta}=2 J_{0} J_{\delta}^{\text {eff }}$.

12 V. N. Kotov, O. Sushkov, Zheng Weihong, J. Oitmaa: Phys. Rev. Lett. 80, 5790 (1998)

13 T. Masuda, A. Zheludev, H. Manaka, L.-P. Regnault, J.-H. Chung, and Y. Qiu, preprint cond-mat/0511143

14 I. A. Zaliznyak, S.-H. Lee, and S. V. Petrov, Phys. Rev. Lett. 87, 017202 (2001). 